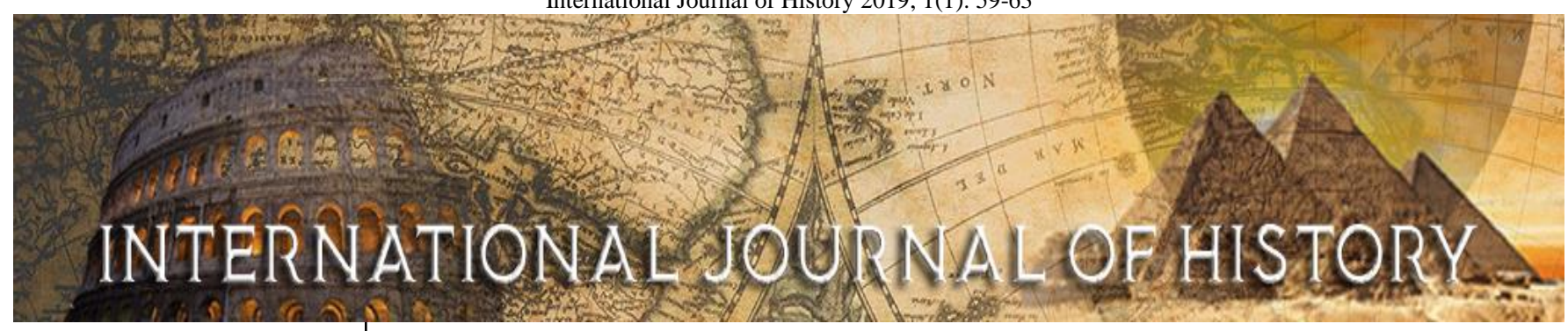

E-ISSN: 2706-9117 P-ISSN: 2706-9109

Impact Factor: RJIF $5.24 \mathrm{~s}$ IJH 2019; 1(1): 59-63

Received: 26-05-2019

Accepted: 18-07-2019

\section{पूनम कुमारी}

इतिहास विभाग, ल० ना० मिथिला विश्वविद्यालय, कामेश्वरनगर, दरभंगा, बिहार, भारत
Corresponding Author: पूनम कुमारी इतिहास विभाग, ल० ना० मिथिला विश्वविद्यालय, कामेश्वरनगर, दरभंगा, बिहार, भारत

\section{आधुनिक भारत में स्वराज के लिए उबलता जनआक्रोश}

\section{पूनम कुमारी}

DOI: https://doi.org/10.22271/27069109.2019.v1.i1a.57

सारांश

युद्ध के वर्षों में भारतीय राष्ट्रवादित को प्रौढ़ता मिली। आंदोलन को गति मिली और एक अर्थ में वह अधिक शक्तिशाली हुआ। ब्रितानी सरकार ने एक सीमा तक युद्ध में भारत के अवदान की प्रशंसा की थी और यह उम्मीद करने का कारण था कि उसे पूर्ण स्वतंत्रता भले न दी जाये, कुछ बड़े सुधार तो किये ही जायेंगे। राष्ट्रवादी नेता भी उम्मीद बांधे हुए थे लेकिन यदि उनकी आशाओं पर पानी फिरता तो वे पुनः संघर्ष के लिए तैयार थे।

19 वीं सदी में भारतीय राष्ट्रीयता की कल्पना के दो आधार रहे हैं- पश्चिमी प्रतिमान के अनुसार अथवा भारतीय सांस्कृति एकता के आधार पर पश्चिमी प्रतिमान का मौलिक आधार राजनीतिक एकता था, जो 19 वीं सदी में पूर्णरूप से स्थापित हुई। भारत में एकता के आधारों भाषा, जाति, धर्म का अभाव दिखाई पड़ा। इसलिए राष्ट्रीयता को भौगोलिक और राजनीतिक आधारों पर स्थापित किया। इस आधार पर भारतीय नेता अंग्रेजी साम्राज्य के प्रति आभारी रहे तथा उसके गुणगान करने और राजनीतिक सुविधाएँ मांगने तक सीमित रहे। राष्ट्रीयता का दूसरा प्रतिमान भारतीय सांस्कृतिक एकता और प्राचीन महानता तथा उपलब्धियों के आधार पर था।

मुख्य शब्द: राष्ट्रवादित, उबलता जनआक्रोश, आधुनिक भारत, 19वीं सदी

\section{प्रस्तावना:-}

आधुनिक भारत में स्वराज के लिए उबलता जनआक्रोश का श्रेय बहुत सीमा तक उन भारतीय विचारकों पर निर्भर रहा, जिन्होंने राष्ट्रीय राजनीतिक आन्दोलन का संचालन किया। सामान्यतः राजनीतिक अधिकारों की चेतना और राष्ट्रीयता की भावना के विकास को पर्यावाची समझा जाता है। लेकिन वास्तव में ऐसा नहीं है, विभिन्न उपनिवेशी में अथवा अधीन राज्यों में राष्ट्रीय आन्दोलन हुए हैं। लेकिन वे आवश्यक रूप से राजनीतिक सुविधाओं और अधिकारों तक सीमित नहीं रहे हैं। राजनीतिक अधिकारों के आन्दोलन भी राष्ट्रीय एवं समस्त देश एवं विदेश वासियों को प्रेरित तथा प्रभावित कर सकते हैं।

सन् 1905 और 1918 के बीच की क्रांतिकारी गतिविधियों की जाँच के लिए रोलेट समिति ने कुछ निश्चित अधिकारियों के लिए सिफारिश की थी। 1905 से 1918 के बीच जनक्रांति को इस लेख में संकलित किया गया है।

\section{मूल शब्द:-}

उग्र राष्ट्रवाद का विकाश, कांग्रेसी संवैधानिक आंदोलन की असफलता, कंजरवेटिव दल की अंकुशल, प्रशासनिक नीति भारत की बढ़ती हुई आर्थिक दरिद्रता, पश्चिमी सर्वोच्चता की कल्पना का अन्त, बंगाल विभाजन : 1905, हिन्दू पुनरूत्थान तथा भारतीय महानता से प्रेरणा, उग्र दल का विकास तथा सूरत फूट : 1907, उग्र दल की कार्य पद्धति, क्रांतिकारी तथा आतंकवादी विचारधारा, विभिन्न प्रान्तों में क्रांतिकारी आंदोलन, विदेशों में क्रांतिकारी आन्दोलन, अमरीका में गदर आन्दोलन, प्रथम विश्वयुद्ध काल में क्रांतिकारी गतिविधियाँ, क्रांतिकारियों की असफलता।

1906-07 में तिलक ने नए उग्र दल के निर्माण तथा गठन में बहुत अधिक सहायता दी। वास्तव में उन्होंने ही सबसे पहले अंग्रेजी साम्राज्य के विरुद्ध शांतिपूर्ण प्रतिरोधक नीति अपनाने की बात कही। तिलक एक ऐसी राष्ट्रीयता को प्रोत्साहन देना चाहते थे, जिनका लक्ष्य राजनीतिक था वह स्वराज्य को भारतीय स्वतंत्रता के लिए आवश्यक समझते थे। 1908-1914 तक तिलक कारावास में रहे। 1914-15 में कुछ प्रयत्न कांग्रेस के दोनों दलों में समझौते के हुए, लेकिन कोई विशेष सफलता नहीं मिली। 1916 में भारतीय रंगमंच पर कुछ विशेष परिस्थितियों का विकास हुआ। मुसलमानों में तुर्की के प्रति अंग्रेजी व्यवहार से असंतोष बढ़ा। अधिकांश प्रभावशाली मुस्लिम नेता भी जेल में थे। भारतीय सेना पर अंग्रेजी निर्भरता बढ़ रही थी और भारत का औद्योगिक तथा मध्यम शिक्षित वर्ग अंग्रेजी 
साम्राज्यवादी नीतियों के दुष्परिणामों से दुखी था वे भी उग्र राष्ट्रीय आंदोलन का समर्थन करने को तैयार थे। अप्रैल, 1916 में तिलक ने होम रूल आंदोलन आरंभ किया। होम रूल अर्थात् स्वराज्य का अभिप्राय इस समय अंग्रेजी साम्राज्य को समाप्त करना नहीं था। इसका अर्थ था अंग्रेजी साम्राज्य के अधीन स्वायत्तता प्राप्त करना। इंग्लैंड के अधीन भारतीय क्षेत्र में भी उसी प्रकार आंतरिक प्रशासन के स्वायत्तता उपलब्ध हो जिस प्रकार भारतीय राज्यों में थी। 1919 में तिलक ने पेरित शांति सम्मेलन में भारत के भाग लेने के अधिकार तथा विल्सन के आत्मनिर्णय के सिद्धान्त के आधार पर भारत के लिए भी इसी अधिकार की मांग प्रस्तुत की। वह खिलाफत आंदोलन के समर्थक थे। तिलक और ऐनिबेसेंट के होम रूल आंदोलन के अभिप्राय का अन्तर दोनों के उस कार्य से स्पष्ट होगा जो 1918 के बाद दोनों नेताओं ने किया। ऐनिबेसेंट भारतीय राजनीतिक रंगमंच से एकदम हट गईं और उन्होंने किसी अन्य राजनीतिक आंदोलन में सक्रिय भाग नहीं लिया।

19 वीं सदी के अंतिम दशक तथा 20 वीं सदी के प्रथम दो दशकों में भारतीय राजनीतिक त्रिमूर्ति - बाल, लाल, पाल - में बाल सबसे अधिक प्रभावशाली रहे। उनके प्रभाव का रहस्य यह था कि उन्होंने अपने आदर्शों को स्वयं साकार किया। उनमें अपने लक्ष्यों के प्रति संघर्ष करने का साहस था। उनके समकालीन अन्य महान नेता - रानाडे, गोखले, सुरेन्द्रनाथ बनर्जी, अरविन्द, विपिनचन्द्र पाल, लाला लाजपतराय आदि थे। तिलक उन सबकी अपेक्षा किसी न किसी अर्थ में उच्चतर थे। रानाडे, गोखले सरकारी प्रश्रय पर परोक्ष या प्रत्यक्ष रूप से निर्भर रहते थे सुरेन्द्रनाथ बनर्जी और फिरोजशाह मेहता पारिवारिक परिस्थितियों से विवश थे। अरविन्द यद्यपि प्रभावशाली वक्ता थे, लेकिन राजनीतिक जीवन के संघर्ष को सहन नहीं कर सके। विपिनचन्द्र पाल अधिक प्रभावशाली वक्ता थे लेकिन राजनीतिक अनुशासन से कम परिचित थे। लाजपतराय की दृष्टि स्पष्ट थी लेकिन उन्हें भी अधिकांश प्रचार कार्य भारत से बाहर जाकर ही करना पड़ा। तिलकने अंग्रेजों के विरुद्ध एक धर्म प्रचारक की भांति उत्साह से कार्य किया। उनके कार्यों को उनके जीवन काल में अंग्रेजों ने जान-बूझकर गलत प्रस्तुत किया। वह उनकी देशभक्ति को राजद्रोह मानते थे। इसलिए उनके सम्बन्ध में बहुत-सी गलत धारणाएँ फैलाते थे।

\section{उग्र राष्ट्रवाद का विकास:-}

19 वीं सदी के अंतिमदशक में इंडिया नेशनल कांग्रेस की लोकप्रियता बहुत कम हो चुकी थी इसका सामान्य जनता पर प्रभाव भी बहुत कम था। वर्ष में एक बार केवल तीन दिन के लिए सम्मिलित होकर कुछ प्रस्तावों के पास कर देने से अधिक इसका कोई कार्य नहीं था। सरकार इसकी मांगों की अनदेखी करती थी, लेकिन 1895-1905 के दशक में कुछ घटनाएँ ऐसी हुई, जिन्होंने न केवल कांग्रेस में एक नया जीवन डाल दिया बल्कि राष्ट्रीय आंदोलन में अंग्रेज विरोधी भावनाओं को प्रोत्साहन दिया। यह प्रोत्साहन इतना अधिक हुआ कि कांग्रेस के दो दल हो गए। अंग्रेज लेखकों ने इस विकास को उग्र राष्ट्रीयता का विकास कहा है। इसमें निम्नलिखित तत्त्व सहायक हुए:-

\section{कांग्रेसी संवैधानिक आंदोलन की असफलता:-}

सरकार से अधिकारों की मांग करने की कांग्रेसी नीति स्पष्ट रूप से असफल दिखाई पड़ी। 1892 के सुधारों में कोई भी वह सिद्धान्त स्वीकार नहीं किया गया जिस पर कांग्रेस ने 1885 से बल दिया था। केवल भारतीय सदस्यों की संख्या मात्र ही बढ़ाई गई थी। अंग्रेजी सरकार की साम्राज्यवादी नीतियों से कोई फेर-बदल नहीं हुआ और न ही प्रजातीय विभेद नीति किसी प्रकार कम होती दिखाई दी। कांग्रेसी आंदोलन की लोकप्रियता भी कम थी। लाला लाजपतराय के अनुसार कांग्रेसी नेताओं के
कुछ राजनीतिक विचार मात्र थे, उनके विश्वास इतने दृढ़ नहीं थे, जिनके लिए वे त्याग अथवा बलिदान कर सकें। इस समय तक त्याग और बलिदान की कल्पना कांग्रेस के अधिकांश राजनीतिक नेताओं में नहीं थी।

\section{कंजरवेटिव दल की अकुशल प्रशासनिक नीति:-}

1888 से 1905 तक इंग्लैंड में कंजरवेटिव दल की सरकार सत्ताधारी रही। इस अवधि में गवर्नर जनरल तथा अंग्रेजी नौकरशाही ने भारतीयों के प्रति अविश्वास तथा प्रजातीय विभेद नीति व्यापक रूप से लाग की। लोकसेवा आयोग की रिपोर्ट ने भारतीयों के लिए आई० सी० एस० में समानता का तर्क स्वीकार नहीं किया और उनके लिए प्रोविंशियल सिविल सर्विस की स्थापना की। 1996-97 के पश्चात् कई ऐसी घटनाएँ हुईं, जिसे प्रशासन का जनता के प्रति क्रूर तथा अमानवीय व्यवहार स्पष्ट हुआ। 1896-97 में बंबई प्रान्त में प्लेग के प्रकोप की रोकथाम के लिए अंग्रेज अधिकारियों का भारतीय जीवन प्रणाली की आलोचना तथा लोगों को बलपूर्वक घरों से निकालकर बाहर करना, दो अंग्रेज अधिकारियों की हत्या के लिए नाटू भाइयों को बिना मुकदमा चलाए जेल में बन्द रखना तथा तिलक पर मुकदमा चलाकर उन्हें दंड देना इस बात के स्पष्ट उदाहरण थे कि अंग्रेज भारतवासियों को प्रशासन के दोषों का ज्ञान उपलब्ध नहीं होने देना चाहते थे। 1899-1901 के मध्य दो बार अकालग्रस्त क्षेत्रों के राहत कार्यों में अंग्रेज अधिकारियों का औपचारिक दृष्टिकोण अत्यंत कठोर था। अंग्रेज भक्त भारतवासियों का अंग्रेजी न्यायप्रियता तथा ईमानदारी पर विश्वास डगमगाने लगा। प्रजातीय विभेद यहाँ तक बढ़ा कि रुड़की इंजीनियरिंग कॉलेज में प्रवेश शुद्ध भारतीयों के लिए मना कर दिया गया। कर्जन की प्रशासनिक नीतियों का परिणाम विस्फोटक हुआ। उच्च शिक्षा पर सरकारी नियंत्रण स्थापित करने, कलकत्ता कारपोरेशन पर सरकारी प्रभाव बढ़ाने, दिल्ली दरबार पर धन का अपव्यय और बंगाल का विभाजन आदि ऐसी घटनाएँ थीं जिन्होंने अंग्रेजी प्रशासन की ईमानदारी और उनके भारतीय शुभाकांक्षी होने में अधिकांश शिक्षित वर्ग का विश्वास समाप्त कर दिया।

\section{भारत की बढ़ती हुई आर्थिक दरिद्रता:-}

1870 के पश्चात् ही कुछभारतीय नेताओं का अंग्रेजी साम्राज्य के आर्थिक परिणामों पर ध्यान आकर्षित होने लगा। धन निष्कासन और आर्थिक शोषण की अंग्रेजी नीति सदी के अन्त तक अधिक नेता समझने लगे थे। भारतीय कुटीर उद्योगों का विनाश, कृषि पर राजस्व का अत्यधिक बोझ, कृषकों की निर्धनता और सरकार की भारतीयों के लिए नौकरी अथवा उद्योग वृद्धि के अवसर उपलब्ध न होने देना ऐसी समस्याएँ थीं, जिनसे यह स्पष्ट हो गया कि अंग्रेजी शासक केवल भारत से धन लूटकर ले जाने को अधिक महत्वपूर्ण मानते थे। 1860 के पश्चात् ही अकालों का प्रकोप बढ़ने लगा। दादाभाई नौरोजी, रमेशचन्द्र दत्त और विलियम डिगवी की पुस्तकों द्वारा अंग्रेजी नीति की वास्तविकता स्पष्ट हो रही थी। वास्तविक आर्थिक स्थिति भी इतनी ही खराब थी। भारतीयों को पूंजी संचय का अवसर ही उपलब्ध नहीं था। प्रभावशाली व्यापारिक प्रतिष्ठान भी अंग्रेजों के नियंत्रण में ही थे। पटसन उद्योग पर उन्हीं का नियंत्रण था। कपड़ा उद्योग भारतीयों के नियंत्रण में अवश्य था, लेकिन उसके विकास मार्ग में अंग्रेजों ने आयात कर समाप्त करके विभिन्न कठिनाइयां खड़ी कर दी थीं। नए मध्यमवर्ग के लिए उचित अवसर नहीं थे। सरकारी सेवाओं का मार्ग उनके लिए प्राय: बन्द ही था। इस व्यापक आर्थिक कठिन परिस्थिति में उग्र विचारों का विकास स्वभाविक ही था।

इन उग्र विचारों के विकास में भारतीय व्यापारियों तथा उद्योगपतियों ने भी सहयोग दिया। इंग्लैंड में बने हुए कपड़े के खुले आयात से और भारत में कपड़ा उद्योग पर लगे उत्पादन 
कर से यहां औद्योगिक विकास बहुत कठिन हो गया थ। कांग्रेस ने भी उद्योगों में पूंजी लगाने के लिए भारतीय पूंजीपतियों को प्रोत्साहन दिया। 19वीं सदी के उत्तरार्द्ध में जापान की आर्थिक प्रगति अत्यधिक प्रशंसनीय तथा अनुकरणीय थी। भारत में यह प्रगति केवल इसलिए नहीं हो सकी थी कि यहां अंग्रेजी नीति का नियंत्रण अत्यधिक था।

\section{पश्चिमी सर्वोच्चता की कल्पना का अन्तः-}

अंग्रेजी इतिहासकारों तथा प्रशासकों ने विभिन्न प्रकार से इस बात पर बल दिया कि भारतीय इतिहास में आक्रांता सदा ही सफल रहे। भारतीय पराजय के लिए यहाँ की सामाजिक, धार्मिक, राजनीतिक, सैनिक दुर्बलताओं पर बल दिया गया। साथ हीजलवायु, जाति, आर्थिक तथा सामाजिक ढ़ांचे के आधार पर विभिन्न प्रकार के मतों के प्रतिपादन पश्चिमी देशों की तुलना में भारतीय हीनता पर ध्यान केन्द्रित करके पश्चिमी सर्वोच्चता की कल्पना व्यापक बनाई गई, जिससे पश्चिमी साम्राज्यवाद सुरक्षित रह सके। 1896 में एबीसीनिया द्वारा इटली को और 1904-05 में जापान द्वारा रूस को पराजित कर दिए जाने के बाद पश्चिमी सर्वोच्चता की यह कल्पना समाप्त हो गई। कुछ भारतीय नेताओं ने जापान की प्रगति का अध्ययन आरंभ किया। जब जापान जैसा छोटा पूर्वी देश रूस जैसे विशाल पश्चिमी देश को हरा सकता था तब भारत भी उन्हीं आधारों पर चलकर बड़ी सरलता से शक्तिशाली बन सकता था, इसलिए भारत की दुर्बलता वास्तविक नहीं थी।

\section{बंगाल विभाजन:-}

अंग्रेजी साम्राज्यवादी नीति के विरुद्ध असंतोष बंगाल विभाजन के पश्चात् बड़ी सरलता से उग्ररूप धारण कर सका। 19 वीं सदी के उत्तरार्द्ध में बंगला भाषी क्षेत्र को मातृभूमि मानने की कल्पना का विकास हुआ। कर्जन द्वारा किया गया बंगाल विभाजन प्रांतीय नेताओं को प्रशासनिक सीमाओं का फेर-बदल न होकर मातृभूति का विभाजन प्रतीत हुआ। इसलिए विभाजन के विरुद्ध सामान्य बंगालियों की भावनाओं को उत्तेजित किया जा सका। बंगाल की नई पीढ़ी के कुछ युवक अंग्रेजी सत्ता के विरुद्ध सशस्त्र विद्रोह की भी योजना बनाने लगी। वहाँ के अधिकांश राजनीतिक नेता निश्चित रूप से प्रार्थना पत्र प्रस्तुत करने को अपर्याप्त समझने लगे और उग्र आंदोलन का सूत्रपात हुआ।

\section{हिन्दू पुनरुत्थान तथा भारतीय महानता से प्रेरणा:-}

राजनीतिक अधिकारों की मांग प्रस्तुत करने वाले अधिकांश नेता पश्चिमी दर्शन, साहित्य, राजनीतिक संस्थाओं की सर्वोच्चता से प्रभावित थे। इसलिए उनका अंग्रेजी साम्राज्य के प्रति दृष्टिकोण विनम्र तथा विनीत था। 19 वीं सदी के अंतिम चतुर्थांश में प्राचीन भारतीय सांस्कृतिक महानता हिन्दुओं की आध्यात्मिक तथा धार्मिक उपब्धियों का ज्ञान भारतीय जनता में फैलने लगा। दयानन्द सरस्वती, स्वामी विवेकानन्द, लोकमान्य तिलक, अरविन्द घोष आदि ने इस भावना को लोकप्रिय बनाया। भारतीय महानता तथा आत्मस्वाभिमान से प्रेरित नेताओं ने अंग्रेजों के प्रति अत्यधिक विनीत दृष्टिकोण न रखकर उग्र विचार पद्धति के विकास में सहायता दी।

\section{उग्र दल का विकास तथा सूरत फूट: 1907:-}

कांग्रेस में उग्र विचारधारा के प्रमुख नेता बालगंगाधर तिलक, लाला लाजपतराय और विपिनचन्द्र पाल थे। 1904 तक उग्र और नरम दल सम्मिलित थे। 1905 में कांग्रेस के बनारस अधिवेशन में सबसे पहली बार इन नेताओं ने अपने अनुयाइयों के साथ अलग बैठक की। लाला लाजपत राय उसी वर्ष इंग्लेंड सरकार को प्रभावित करने में असमर्थ लौटे। बंगाल विभाजन अक्तूबर, 1905 में किया गया। फलस्वरूप कांग्रेस अधिवेशन में सविनय प्रार्थना
की पुरानी नीति से असंतुष्ट सदस्यों की संख्या बढ़ती दिखाई दी। स्वदेशी और बायकाट के प्रश्नों पर मतभेद स्पष्ट था। 1906 में प्रिंस ऑफ वेल्स के भारत आगमन स्वागत से सम्बन्धित प्रस्ताव कुछ सदस्यों की अनुपस्थिति में ही पास किया जा सका। तिलक चाहते थे कि सरकार के विरुद्ध शांतिपूर्ण प्रतिरोधक नीति के सम्बन्ध में एक प्रस्ताव पास किया जाए लेकिन ऐसा न हो सका। फलस्वरूप 1905 की समाप्ति पर इन दो विभिन्न विचारधाराओं के अनुयाइयों में मनमुटाव बढ़ता दिखाई पड़ा। 1906 में कलकत्ता कांग्रेस अधिवेशन उग्र पक्ष के लिए शक्ति परीक्षा स्थल बन गया। वे तिलक को कांग्रेस अध्यक्ष बनाना चाहते थे, जिसे संवैधानिक नीति के समर्थक स्वीकार नहीं कर सकते थे। अन्त में वृद्ध दादा भाई नौरोजी को अध्यक्ष बनाने पर दोनों सहमत हो गए। 1905 में इंग्लेंडमें लिबरल दल को निर्वाचन में बहुत मिल जाने से संवैधानिक पक्ष को अपने कार्यक्रम के सफल होने की अंतिम आशा दिखाई पड़ी। 1906 में बंगाल में स्वदेशी और बायकाट (विदेशी कपड़े का बहिष्कार) की नीति जोरों पर रही। कांग्रेस के कलकत्ता अधिवेशन (1906) में उग्र दल की समस्त नीति अत्यधिक वाद-विवाद के पश्चात् स्वीकृत हुई। 'स्वराज्य', 'स्वदेशी', 'बायकाट' और 'राष्ट्रीय शिक्षा' से सम्बन्धित चार प्रस्ताव पास किए गए। संवैधानिक मत वाले लोग प्रस्ताव पास कर देना मात्र ही पर्याप्त समझते थे। वे इस दिशा में कोई अन्य कार्य नहीं करना चाहते थे। इसलिए उग्र पक्ष का नीति का अनुमोदन भी संतोषप्रद नहीं था दूसरी ओर मिन्टो ने उग्र पक्ष को प्रभावशाली न बनने देने के लिए भावी सुधारों के विषय में 'संवैधानिक' पक्ष के नेताओं के विचार-विमर्श आरंभ किया। मोर्ले और गोखले में एक प्रकार का समझौता इंग्लैंड में हो चुका था। उग्र पक्ष का संवैधानिक पक्ष पर संदेह स्वाभाविक ही था इसलिए 1907 में कांग्रेस संगठन पर दोनों पक्षों ने अपना प्रभुत्व स्थापित करने का प्रयत्न किया। संवैधानिक पक्ष नें 1906 में पास किए गए प्रस्तावों से पीछे हटना चाहा। इस प्रकार 1907 में आयोजित कांग्रेस का सूरत अधिवेशन महत्वपूर्ण सिद्ध हुआ।

सूरत में कांग्रेस फूट का मूल कारण यह था कि नरम दल के नेता 1906 में पास किए गए प्रस्तावों को लागू नहीं करना चाहते थे। संभवतः इसके पीछे अंग्रेज सरकार का हाथ था, जिससे उग्र पक्ष कांग्रेस से अलग हो जाए। अधिवेशन आरंभ होने के पूर्व तिलक ने अलग संगठन बनाने का सुझाव अस्वीकृत कर दिया। वह 1906 में पास किए हुए कार्यक्रम को लागू करना अथवा आगे बढ़ाना चाहते थे। 1907 के लिए लाजपतराय के स्थान पर रासबिहारी घोष को अध्यक्ष बनाना ही उग्र दल के लिए आपत्ति का विषय था। वह लाला लाजतपराय को अध्यक्ष बनाना चाहते थे। अधिवेशन आरंभ होने के पश्चात् तिलक ने मंच पर आकर निर्वाचन के सम्बन्ध में कुछ आपत्तियाँ उठानी चाहीं लेकिन उन्हें बोलने की अनुमति नदीं दी गई। इस अवसर पर श्रोताओं में से किसी ने एक जूता सुरेन्द्रनाथ बनर्जी पर फेंका और फिर पंडाल में अव्यवस्था फैल गई। कुर्सी और मेजों को एक दूसरे पर फेंका गया। कांग्रेस अधिवेशन स्थगित कर दिया गया। यह घटना 24 दिसम्बर, 1907 की थी।

इस घटना के बाद उग्र दल के अनुयाइयों को कांग्रेस से बाहर होना पड़ा। कांग्रेस नरम दल के हाथों में रही। सूरत फूट के लिए सामान्यतः उग्र दल के अनुयाइयों को दोषी ठहराया जाता है लेकिन वास्तव में ऐसा नहीं था। स्वयं रासबिहारी घोष ने अपने अपठित अध्यक्षीय भाषण में उग्र विचारों के सदस्यों को कांग्रेस से बाहर जाने का परामर्श दिया था। उग्र विचारों वाले सदस्यों को कांग्रेस से निकल जाने पर विवश किया गया।

\section{उग्र दल की कार्य पद्धति:-}

उग्र दल का विकास उदारवाद के विरुद्ध हुआ। इसलिए उसका संवैधानिक प्रार्थना पत्रों की उपयोगिता में विश्वास नहीं था। वह अंग्रेजी साम्राज्य को नैतिकता अथवा न्याय पर आधारित नहीं 
समझता था, इसलिए नैतिक तर्कों का प्रयोग उसे प्रभावित करने में निरर्थक अथवा व्यर्थ था। संवैधानिक तथा नरम नीति के समर्थक उस समय अंग्रेजी साम्राज्य के बाहर भारत की कल्पना नहीं करते थे। वे अपनी योग्यता का अंग्रेजों को विश्वास दिलाकर उनसे सुविधाएं चाहते थे। उग्र दल के नेता समझते थे कि अंग्रेजी साम्राज्य से सहयोग नैतिक तथा आर्थिक दृष्टि से भारत के लिए हानिकारक था।

संवैधानिक दल की भांति वे अपने कार्य को वर्ष में केवल तीन या चार दिनों तक ही सीमित नहीं रखना चाहते थे। उनका लक्ष्य वर्ष भर प्रभावशाली ढंग से आंदोलन करते रहना था। सामान्य जनता तथा समस्त राष्ट्र को प्रभावशाली आंदोलन से इस प्रकार गठित तथा जाग्रत किया जाए, उसमें स्वतंत्रता के प्रति ऐसी बलिदान की भावना पैदा की जाए कि शासकों को विवश होकर उसके समक्ष झुकना पड़े। वे अहिंसात्मक प्रतिरोधक (सत्याग्रह) से उस नैतिक प्रभुत्व को तोड़ना चाहते थे जो अंग्रेज साम्राज्यवादियों द्वारा भारतीयों के ऊपर स्थापित किया हुआ था $2^{2}$ वे स्वतंत्रता के प्रति गहरा लगाव पैदा करके देश के लिए त्याग तथा बलिदान की भावना जाग्रत करना चाहते थे, उनका कार्यक्रम केवल सत्याग्रह तक ही सीमित नहीं था बल्कि रचनात्मक भी था। वे स्वदेशी (भारतीय वस्तुओं के प्रयोग) को प्रोत्साहन देना तथा विदेशी वस्तुओं का वायकाट करना चाहते थे। वे सरकारी शिक्षा पद्धति को छोड़कर एक अलग राष्ट्रीय शिक्षा पद्धति की स्थापना करना चाहते थे, और यदि संभव हो सके तो एक पृथक् प्रशासन पद्धति भी स्थापित की जाए, जिससे सरकार पर निर्भरता कम हो सके। उनकी यह पृथक् योजना सरकार के विरुद्ध नहीं थी, बल्कि सरकार के नियंत्रण से मुक्त थी यह मुक्ति ही सरकार के लिए असह्य थी। उग्र दल ने राष्ट्रीय संघर्ष के लिए एक नया लक्ष्य 'स्वराज्य' प्रदान किया।

\section{क्रांतिकारी तथा आतंकवादी विचारधारा:-}

उग्र राष्ट्रवादियों में एक वर्ग ऐसा भी था जो बलपूर्वकअंग्रेजी साम्राज्य को समाप्त करना चाहता था। इस वर्ग में भिन्न-भिन्न विचारों वाले कई समूह सम्मिलित थे। यह वर्ग अंग्रेजी साम्राज्य को बल तथा धोखे पर आधारित समझता था, इसलिए बलपूर्वक षड्यंत्र अथवा सशस्त्र विद्रोह से उसे समाप्त करना उचित मानता था। इस वर्ग के कुछ अन्य क्रांतिकारी अंग्रेजी प्रशासन को हिन्दू धर्म तथा भारत भूमि के विरुद्ध मानते थे। इन विचारकों के समर्थक बंगाल में अधिक थे और बंगाल विभाजन को अपनी मातृभूमि का विभाजन मानते थे। यह वर्ग अंग्रेजी अधिकारियों ही हत्या तथा सरकारी खजाने की लूटमार को अपराध नहीं समझता था। एक अन्य वर्ग अंग्रेजी साम्राज्य का तख्ता ही पलट देना चाहता था। सेना में अंग्रेज विरोधी प्रचार अथवा विद्रोह एवं संगठित प्रयत्नों द्वारा वे अपना लक्ष्य प्राप्त करना चाहते थे। कुछ लोग स्वतंत्रता प्रेम से इतना अधिक प्रभावित थे कि वे किसी भी प्रकार के प्रयत्नों को उचित मानते थे।

\section{विभिन्न प्रांतों में क्रांतिकारी आन्दोलनः-}

यह आंदोलन मख्यतः तीन प्रांतों में केन्द्रित रहा - बंगाल, महाराष्ट्र तथा पंजाब। बंगाल में यह 1905 के विभाजन के पश्चात् आरंभ हुआ। वीरेन्द्र कुमार घोष और भूपेन्द्र दत्त ने 'युगांतर' पत्रिका की स्थापना की। यह पत्र सबसे अधिक क्रांति का प्रचारक था। इसी प्रकार की अन्य पत्रिका 'संध्या' व 'नवशक्ति' थी। अरविन्द ने जोशीले लेखों द्वारा राष्ट्रीय प्रयत्नों को अधिक प्रोत्साहन दिया। उनके लेख 'वंदे मातरम' में प्रकाशित होते थे। उन्हें अलीपुर बम मुकदमे के सम्बन्ध में बंदी बना लिया गया। बंदी बना लेने से उनके उग्र विचारों में कोई परिवर्तन नहीं आया। इतना अवश्य हुआ कि वह राजनीति से अलग हटकर धार्मिक और सांस्कृति एकता का प्रचार करने लगे। बंगाल के बहुत से नवयुवक फ्रांस और रूस जाकर बम बनाने की विधि
सीखने लगे। अक्तूबर, 1907 में बंगाल के गवर्नर की गाड़ी को बम से उड़ाने का असफल प्रयास किया गया। अलीपुर बम मुकदमे में सरकारी प्रोसीक्यूटर और पुलिस के डिप्टी सुपरिटेंडेंट की हत्या कर दी गई। वरींद्र और उनके सहयोगियों का कार्य 'अनुशीलन समिति' के द्वारा चलाया जाता रहा। 1907 से 1917 तक 60 व्यक्तियों से अधिक की हत्या कर दी गई। 100 से अधिक राजनीतिक डकैतियों में सरकारी खजाने अथवा साम्राज्य समर्थक व्यक्तियों को लूटने के प्रयत्न किए गए। हथियारों और बमों को विदेशों से मंगाया गया। इसी प्रकार की क्रांतिकारी संस्थाएँ तथा घटनाएँ बिहार, उड़ीसा, पंजाब, महाराष्ट्र तथा भारत के अन्य भागों में भी हुईं।

\section{विदेशों में क्रांतिकारी आन्दोलन:-}

अंग्रेजी साम्राज्य के निरंकुश नियंत्रण से बचने के लिए क्रांतिकारियों को बहुत बार भारतीय स्वतंत्रता के लिए अपने प्रयत्नों का मुख्य कार्यालय अन्य देशों में स्थापित करना पड़ा। सबसे पहले लन्दन में श्री श्यामजी कृष्ण बर्मा ने फरवरी, 1905 में इण्डियन होम रूल सोसाइटी की स्थापना की। उन्होंने साहसी भारतीय युवकों को आर्थिक सहायता देकर विदेशों में भ्रमण तथा अनुभव अर्जित करने की प्रेरणा दी, हरदयाल, मदन लाल ढींगरा, विनायक दामोदर सवारकर आदि उनके सहयोगी बने। श्यामजी को अपना कार्यालय शीघ्र ही लंदन से हटाना पड़ा।

\section{अमरीका में गदर आंदोलन:-}

19 वीं सदी केअन्तिम दशक में पंजाब के किसान काफी संख्या में संयुक्त राज्य अमरीका में जाकर रहने लगे। वहाँ 2-3 डालर प्रतिदिन मजदूरी मिल जाती थी। भारतीय श्रमिक अपेक्षाकृत सस्ते होने के कारण अमरीकी पूंजीपति उनकी सहायता से अमरीकी मजदूर संगठनों के नियंत्रण से मुक्त हो जाते थे। किन्तु वे उसी कारण सामान्य अमरीकी दृष्टि में अपमानित समझे जाते थे। अमरीका में रहने वाले भारतीय स्वराज्य और स्वदेशी भावनाओं से परिचित थे। अमरीका में उन्हें प्रचार की स्वतन्त्रता थी इसलिए अंग्रेजी अत्याचारों तथा निरंकुश नौकरशाही के विरुद्ध अमरीका में प्रचार आरंभ हुआ। 1913 में विभिन्न भारतीयों ने मिलकर सेन फ्रांसिस्को में 1 नवम्बर, 1913 के गदर पार्टी की स्थापना की और एक साप्ताहिक (बाद में मासिक) पत्रिका 'गदर' का प्रकाशन आरम्भ किया। लाला हरदयाल इसके मुख्य कार्य संचालक थे। इस पत्रिका में अंग्रेजी अन्याय और अत्याचार का खुला वर्णन होता था। इससे एक ओर अंग्रेजी के विरुद्ध प्रचार होता था तथा दूसरी ओर भारतीय नागरिकों को स्वतन्त्रता प्राप्त करने के लिए प्रेरणा मिलती थी। भारत में अंग्रेजी अत्याचारों का अन्तर्राष्ट्रीय प्रचार इसके माध्यम से हुआ।

\section{प्रथम विश्वयुद्ध काल में क्रांतिकारी गतिविधियाँ:-}

क्रांतिकारियों को प्रथम विश्वयुद्ध एक स्वर्ण अवसर प्रतीत हुआ। उन्हें जर्मनी (इंग्लैंड के शत्तु) से सहायता की आशा प्रतीत हुई। इंग्लेंड की कठिनाई से लाभ उठाने का भी यह अच्छा अवसर था। जर्मनी भी सहायता के लिए उत्सुक था, क्योंकि भारत के विद्रोह का इंग्लैंड की सैनिक शक्ति पर प्रभाव निश्चित ही था। उसका दृष्टिकोण भारतीय आंदोलन के प्रति सहानुभूति न होकर इंग्लैंड से शत्रुता का परिणाम था। जर्मनी में एक 'इंडियन इंडिपेंडेंस कमेटी' की स्थापना की गई। भारतीय क्रांतिकारियों को अस्त्र-शस्त्र पहुंचाना तथा आर्थिक सहायता देना इस संस्था का मुख्य कार्य था। अमरीका में गदर पार्टी भी काफी प्रयत्नशील रही।

बंगाल विभाजन के 1911 में रद्द कर दिए जाने के पश्चात् वहाँ क्रांतिकारी घटनाएं बहुत कम हो गई। तुर्की के युद्ध में अंग्रेजों के विरुद्ध हो जाने तथा तुर्की के सुल्तान द्वारा खिलाफत की सुरक्षा के लिए मुसलमानों से सहायता की प्रार्थना करने पर तथा 
पंजाबियों के अमरीका से लौटने पर अंग्रेज विरोधी भावनाएँ वहाँ अत्यधिक व्यापक हुई। मौलवी उबैदुल्ला सिंधी, मौलाना महमूद हसन का योगदान उल्लेखनीय था। उबैदुल्ला द्वारा कुछ पत्र महसूद हसन को लिखे गए। ये पत्र पीली सिल्क पर फारसी में लिखे गए और पकड़े जाने पर 'सिल्क पेपर्स षड्यंत्र' समाप्त हुआ। इसी प्रकार अमरीका से लौटे हुए पंजाबियों ने भी सशक्त विद्रोह की योजना बनाई लेकिन एक कार्यकर्त्ता ने गुप्त सूचना दे दी और वह षड्यंत्र भी अधूरा ही रह गया।

\section{क्रांतिकारियों की असफलता:-}

सशस्त्र प्रयत्नों अथवा बलपूर्वक अंग्रेजी साम्राज्य को पलटने के भारतीय क्रांतिकारियों के प्रयत्न सामान्यतः असफल रहे। इन प्रयत्नों की असफलता के कई कारण थे। भारतीय समुदाय के अत्यन्त छोटे वर्ग ने ही इस प्रकार के प्रयत्नों का समर्थन किया। अधिकांश प्रतिष्ठित व्यक्तियों ने इस प्रकार के प्रयत्नों को आलोचनात्मक दृष्टि से देखा। विभिन्न प्रांतों के क्रांतिकारियों ने कोई संगठित प्रयत्न नहीं किए। सरकार ने दमनकारी नीति, खुफिया विभाग तथा क्रांतिकारियों में से ही किसी एक ही सहायता से विभिन्न प्रयत्नों को समय से पूर्व समाप्त कर दिया।

\section{निष्कर्ष:-}

अंग्रेज इतिहासकारों, कांग्रस समर्थकों तथा प्रतिष्ठित वर्गों की व्यापक आलोचना के कारण क्रांतिकारियों के प्रयत्नों का महत्व ठीक तरह से नहीं आंका जाता है। व्यक्तिगत प्रयत्नों की निरर्थकता, युवक वर्गों का भावनाओं से प्रेरित होना तथा सशस्त्र विद्रोह का अनैतिक होना आदि तर्क प्रस्तुत करके इन क्रांतिकारियों के योगदान को नगण्य बताया जाता है। इसमें संदेह नहीं कि क्रांतिकारी अपने लक्ष्य को प्राप्त करने में असफल रहे, गांधीजी के नेतृत्व में अहिंसात्मक आंदोलन की कालांतर में सफलता से सशस्त्र प्रयत्नों की और भी अधिक आलोचना होती रही है, लेकिन कुछ तथ्य ध्यान में रखने चाहिए। 1906 के पश्चात् अंग्रेज सरकार ने कांग्रेसी तथा अन्य नेताओं की बात को अपेक्षाकृत अधिक ध्यान से सुना, यह इसीलिए संभव हो सका, क्योंकि अंग्रेजी सशस्त्र विद्रोह की भावना तथा आंदोलनकी वृद्धि को रोकना चाहते थे। दूसरे राष्ट्रीय स्मृति में शहीदों का स्थान उन लोगों को प्राप्त हुआ जिन्होंने अंग्रेजों के विरुद्ध असफल सशस्त्र प्रयास किया। प्रसिद्ध भारतीय नेताओं- तिलक, अरविन्द, लाजपतराय, अश्विनी कुमार दत्त- ने प्रत्यक्ष अथवा परोक्ष रूप से क्रांतिकारियों का समर्थन किया। आयरलैंड, इटली, रूस में व्यक्तिगत तथा समुदायगत सशस्त्र प्रयत्न विदेशी अथवा निरंकुश सत्ता के विरुद्ध किए गए। इन क्रांतिकारियों ने देश प्रेम के लिए त्याग और बलिदान का उज्ज्वल उदाहरण जनता के समक्ष प्रस्तुत किया। त्याग की यह भावना ही अन्ततः अहिंसात्मक आंदोलन की सफलता के लिए उत्तरदाई हुई। स्वयं इंग्लैंड में भी सशस्त्र विद्रोह और गृहयुद्ध के पश्चात् ही 1688 की रक्तहीन क्रांति संभव हो सकी थी। 1832 के सुधार अधिनियम के पूर्व भी इंग्लेंड में विद्रोह की संभावना थी। इसलिए सशस्त्र प्रयत्नों को निष्फल कहना ठीक नहीं है।

उग्र दल के तीन प्रमुख नेता थे जो लाल-बाल-पाल की त्रिमूर्ति से जाने जाते हैं। बालगंगाधर तिलक के सम्बन्ध में हम पहले ही वर्णन कर चुके हैं। वह अपनी लोकप्रियता के कारण लोकमान्य कहलाए गए। दूसरे प्रमुख नेता लाला लाजपतराय, विपिनचन्द्र पाल थे।

\section{संदर्भ ग्रन्थों की सूची:-}

1. पी० सी० घोष :- इडियन नेशनल काँग्रेस 1892 - 1909, पृ०-196.

2. लाजपतराय : यंग इडिया; पृ०-141-142.

3. अर्गो, पृ०-195 : यह भाषण दिसम्बर, 1928 ई0 के 'मोडर्न
रिव्यू में दिया है, पृ०-76.

4. बिपनचन्द्र, अमलेश त्रिपाठी, बरूण दे : स्वतंत्रता संग्राम, पृ0-91-94.

5. डा० आर० के० परन्थी : आधुनिक भारत (1858-1905) संगठित राष्ट्रवाद का उदय, पृ०-143 से 163.

6. एस० गोपाल : 'ब्रिटिश पालिसी इन इंडिया'।

7. एस0 गोपाल : 'दि वयासरोयल्टी ऑफ लार्ड रिपन'।

8. एस० गोपाल : 'ब्रिटिश पॉलिसी।

9. डोडवेल : 'कैब्रिज हिस्ट्री ऑफ इंडिया'।

10. डी० के० घोष : इंग्लैंड एण्ड अफगानिस्तान।

11. विशेश्वर प्रसाद : फाउन्डेशंस ऑफ इंडियाज फारेन पालिसी, पृ0-230-231.

12. मेटकाफ 'दी आक्टरमैथ ऑफ रियोल्ट : पृ०- 98 